\title{
ATIVIDADE ELETROMIOGRÁFICA DA MUSCULATURA DO QUADRÍCEPS \\ ASSOCIADO ÀS DIFERENTES POSIÇÕES DO PÉ: UM ESTUDO PILOTO
}

\author{
ELECTROMYOGRAPHIC ACTIVITY OF THE QUADRICEPS MUSCULATURE \\ ASSOCIATED WITH THE DIFFERENT FUNCTIONS OF THE FOOT: A PILOT \\ STUDY
}

\section{ACTIVIDAD ELECTROMIOGRAFÍCA DE LOS MÚSCULOS DEL CUÁDRICEPS ASOCIADO A LAS DIFERENTES POSICIONES DEL PIE: UN ESTUDIO PILOTO}

\author{
Poliana Lima Costa Loures ${ }^{2}$, Francielle Pereira Santos 1,2, Luiz Gustavo Monteiro ${ }^{1}$, \\ Cintia Campolina Duarte Rocha da Silva ${ }^{2}$ e Sandro Fernandes da Silva ${ }^{1}$
}

poli.edf@hotmail.com; franpereiras@hotmail.com; gustavomonteiro76@globomail.com; cintiacdrs@gmail.com; sandrofs@ufla.br

\author{
1 Universidade Federal de Lavras, Brasil \\ ${ }^{2}$ Faculdade Presbiteriana Gammon, Brasil
}

Envio original: 2020-01-02 Reenviado: 2020-08-07Aceitado: 2020-08-25

Publicado: 2020-09-08

Doi: https://doi.org/10.15517/pensarmov.v18i2.40268

\begin{abstract}
RESUMO
O fortalecimento do grupo muscular quadríceps femoral (QF) é muito importante devido sua função para o movimento. Mudanças na posição do pé, podem gerar alterações no recrutamento muscular, desta forma o presente estudo objetivou verificar se as variações nas posições dos pés, durante a extensão de joelhos, são eficazes para a seleção específica dos músculos reto femoral, vasto lateral e vasto medial, que compõem o QF. Onze mulheres universitárias (idade 22,36 \pm 3,56 anos) participaram do estudo. Foi avaliada a ativação dos músculos através da eletromiografia de superfície, com configuração bipolar, em 3 séries de 10 repetições máximas (RM) de extensão de joelhos bilateral, em três posições dos pés: posição neutra, rotação interna e rotação externa. Os resultados não apontam diferenças significativas entre os músculos do QF superficial em todas as posições dos pés. Em conclusão, os resultados do presente estudo sugerem que as variações nos graus de rotação dos pés durante a
\end{abstract}


extensão de joelhos, não são eficazes para a seleção específica dos músculos que compõem o QF.

Palavras-chave: avaliação eletromiográfica, extensores de joelho, posição dos pés

\begin{abstract}
The strengthening of the femoral quadriceps (FQ) muscular group is very important due to its function for moving. The joint position might generate modifications in muscular recruitment, therefore the objective of this study was to verify if the feet positions variations during knee extension, are efficient for the specific selection of the rectus femoris, vastus lateralis and vastus medialis muscles, that compose the FQ. Eleven female college students (age 22,36 $\pm 3,56$ years old) participated in the study. The muscles activation was evaluated through surface electromyography, with bipolar configuration, in 3 series of 10 maximum repetition (MR) of bilateral knee extension, in three feet positions: neutral position, internal rotation and external rotation. The results do not indicate significant differences among the superficial FQ muscles in all feet positions. In conclusion, the results of the present study suggest that the variations in degrees of feet rotation during knee extension are not efficient for the specific selection of the muscles that compose the FQ.
\end{abstract}

Key words: electromyographic evaluation, knee extensor, feet variation

\title{
RESUMEN
}

El fortalecimiento de los músculos del cuádriceps femoral (CF) es importante debido a su función en el movimiento. La posición de la articulación puede generar cambios en el reclutamiento muscular, de esa forma el presente estudio tiene como objetivo verificar si variaciones en las posiciones del pie durante la extensión de las rodillas son eficaces para la selección especifica de los músculos recto femoral, vasto lateral y vasto medial, que componen el CF. Once mujeres universitarias (edad 22,36 $\pm 3,56$ años) participaron del estudio. Fue evaluada la activación de los músculos a través de la electromiografía de superficie, con una configuración bipolar, en tres posiciones de los pies: neutra, rotación interna y rotación externa. Los resultados no presentaron diferencias significativas entre los músculos del CF en todas las posiciones del pie. En conclusión, los resultados del presente estudio sugieren que las variaciones en los grados de rotación de los pies durante la extensión de las rodillas no son eficaces para la selección específica de los músculos que componen el CF. 
Palabras clave: evaluación electromiografíca, extensores de la rodilla, variaciones del pie.

\section{INTRODUÇÃO}

O quadríceps femoral (QF) é um grupo muscular situado na face anterior da coxa, constituído pelos músculos reto femoral (RF), vasto lateral (VL), vasto medial $(V M)$ e vasto intermédio (VI) (Ando et al., 2016), que agem de forma integrada para gerar torque extensor no joelho (de Souza, Cabral, de Oliveira y Vieira, 2018; Spairani et al.,2012). O QF tem sido amplamente investigado na literatura (Akima \& Ando,_2017; Ando, Tomita, Watanabe \& Akima, 2018; Wu, Delahunt, Ditroilo, Lowery \& De Vito, 2017), por exercer papeis importantes no movimento humano, como caminhar, correr e subir escadas (Saito \& Akima, 2015; Watanabe \& Akima, 2009) e, por atuarem como os principais estabilizadores dinâmicos da patela (Lanza, Balshaw \& Folland, 2017).

Neste sentido, (Briani et al., 2018; Saito \& Akima, 2015), afirmam que um desequilíbrio gerado nos músculos VM e VL podem comprometer a cinemática patelar, contribuindo para o aumento das forças de reação e compressão femoropatelares. Estas ações podem gerar tensão excessiva nas estruturas citadas, podendo desencadear um quadro de dor e mau alinhamento patelar (Fagan \& Delahunt, 2008; Noorkõiv, Nosaka \& Blazevich, 2014). Sintomas como estes, podem estar relacionados a síndrome da dor femoropatelar, patologia que acomete atletas e não atletas e que representa um problema comum no joelho de adolescentes e adultos jovens fisicamente ativos (Ando et al., 2018; Fagan \& Delahunt, 2008).

Segundo, (Akima, Tomita \& Ando, 2019; Watanabe \& Akima, 2009), a orientação variada do pé no plano sagital, altera as cargas do tornozelo, joelho e quadril, pela mudança na postura da perna e direção resultante do vetor de força. Spairani et al., (2012), declaram que em uma ação muscular isométrica do QF, tanto a posição do pé, quanto o grau de extensão do joelho, influenciam na ativação eletromiográfica dos músculos do QF. Estes mesmos autores, em um estudo clássico em 1995, analisaram as diferentes posições do pé (posição neutra ( $P N)$, rotação externa (RE) e rotação interna (RI) no exercício dinâmico de cadeira extensora, e observaram que a RE produz maiores ativações em todos os músculos do QF, sendo o RF o músculo mais ativado (Signorile, Kwiatkowski, Caruso \& Robertson, 1995). Já, Stoutenberg, Pluchino, Ma, Hoctor e Signorile (2005), encontraram em seus resultados, uma maior ativação eletromiográfica na RE no músculo RF, entretanto na posição RI, identificou-se maior ativação do VL e do VM.

Sabendo que as diferentes posições e ângulos dos pés durante um exercício pode alterar a ativação muscular do QF (Lanza et al., 2017; Murray, Cipriani, O’Rand \& Reed- 
Jones, $\underline{2013}$ ), e que fatores como as variações na orientação da fibra muscular e a ligação da patela, resultam em diferenças marcantes na direção dos vetores de força e na funcionalidade do QF (Cha, 2014), reforça-se a importância de se conhecer as alterações provocadas pelas mudanças da posição dos pés na ativação das musculaturas do QF.

Diante do exposto, apresenta-se o objetivo do presente estudo, que foi verificar a interferência da utilização de diferentes posições dos pés durante o movimento de extensão de joelhos, na seleção específica dos músculos que compõem o QF. Esperase encontrar (a) maior ativação do músculo RF durante a PN; (b) maior ativação do VL durante a Rl; e, maior ativação do VM em RE, durante o exercício de cadeira extensora.

\section{MATERIAIS E MÉTODOS}

\section{Sujeitos}

A seleção da amostra foi intencional, considerando um tamanho do efeito $(f)=0,8$; poder $(1-\beta)=0,5 ; \alpha=0,05$, perfazendo um total de onze mulheres universitárias (idade $22,36 \pm 3,56$ anos, massa corporal 59,48 $\pm 5,88 \mathrm{~kg}$, estatura 164,20 \pm 4,63 cm, percentual de gordura $23,10 \pm 3,26 \%$ ), saudáveis, fisicamente ativas, com experiência prévia no treinamento resistido e na execução do movimento de cadeira extensora. As participantes não apresentavam histórico de lesões e/ou cirurgias musculoesqueléticas que impossibilitaram a realização de exercício de extensão de joelho extenuante. Os procedimentos do estudo foram aprovados pelo Comitê de Ética 01565412.0.0000.5148 e, estavam de acordo com os regulamentos estabelecidos pelos responsáveis da Comissão de Investigação Clínica e Ética e de acordo com a Associação Médica Mundial e da Declaração de Helsinki.

\section{Desenho Metodológico}

Para o início do estudo, após as avaliações antropométricas, os sujeitos foram submetidos a um teste para determinar a carga máxima relativa a 10-RM durante a extensão de joelhos, bilateral, na cadeira extensora (Physicus $₫$ Linha Pro).

Após um período de 48 horas, foi aplicado o teste que consistiu, primeiramente, em um aquecimento de 20 repetições a $50 \%$ de 10-RM e um intervalo de 2 minutos. Posteriormente, foram realizadas 3 séries de 10-RM de extensão de joelhos bilateral, sendo cada série executada em uma posição do pé (PN, RI e RE), com intervalo de 5 minutos entre elas.

As ativações eletromiográficas foram coletadas unicamente nos músculos da perna direita, sendo mantida uma duração 4 segundos em cada movimento (2 segundos 
para a fase concêntrica e 2 segundos para a fase excêntrica, controlados a partir de um metrônomo). Vale ressaltar que em toda a coleta, foram seguidas as recomendações do Surface Electromyography for the Non-Invasive Assessment of Muscle (SENIAN) (Hermens, Merletti \& Freiks, 1996).

As variações da posição dos pés para a avaliação do músculo QF foram (a) posição neutra (PN), na qual as pernas ficavam alinhadas ao quadril e não há rotações dos pés; (b) rotação interna (RI), na qual a articulação do quadril sofria uma ligeira rotação interna, favorecendo esta rotação também ao joelho e aos pés; (c) rotação externa (RE), na qual a articulação do quadril sofria ligeira rotação externa, favorecendo a mesma rotação aos joelhos e aos pés.

Em situações práticas, o ângulo de RI ou RE dos pés depende da amplitude de movimento e da capacidade do indivíduo de manter a rotação durante todo o movimento de extensão de perna, não sendo necessário a determinação de ângulos específicos para cada posição dos pés.

A ordem das variações das posições dos pés foi executada aleatoriamente para cada indivíduo, visando minimizar os efeitos de fadiga e outras possíveis alterações decorrentes da sequência dos exercícios.

\section{Instrumentos}

\section{A) Eletromiografia}

O sinal eletromiográfico foi analisado durante todo o movimento de cada exercício de extensão de joelhos nas diferentes posições dos pés. Os músculos do quadríceps RF, VM e VL - foram avaliados através da eletromiografia de superfície (Miotool 400 Miotec Equipamentos Biomédicos Ltda, POA, Brasil(), com configuração bipolar, para maximizar a área de recepção dos sinais, sendo a fixação dos pares de eletrodos em pontos propostos pela SENIAN (Hermens, et al., 1996), paralelos às fibras musculares ativas, de acordo com a penação de cada músculo. Um eletrodo de referência foi colocado sobre a patela. A preparação do local de fixação dos eletrodos nos músculos consistiu na remoção dos pelos, abrasão e limpeza da epiderme da perna direita para a minimização de possíveis interferências que reduzem a fidelidade dos sinais.

Para a análise das ativações eletromiográficas, os sinais foram filtrados utilizando o filtro Butterworth de $5^{\mathrm{a}}$ ordem, do tipo passa banda, com frequência de corte de 20 $500 \mathrm{~Hz}$, para eliminação de possíveis riscos de sinais. Assim, determinou-se o valor médio e máximo das ativações, utilizando o RMS (Root Mean Square). O software Miograph 2.0 Alpha 9 Build 5, foi utilizado para análise e processamento dos dados. 


\section{Análise Estatística}

Utilizou-se a estatística descritiva com comparação de médias e desvio padrão. Para verificar a distribuição da amostra foi adotado o teste de Shapiro-Wilk. Como a distribuição foi não homogênea, utilizou-se o teste de Friedman para analisar as ativações eletromiográficas entre as diferentes posições dos pés, e as ativações nos grupos musculares estudados (RF, VM e VL), em relação à cada posição dos pés. $\mathrm{O}$ software utilizado para todas as análises foi o IBM SPSS Statistics 20.0, e para significância estatística foi adotado $p \leq 0,05$

\section{RESULTADOS}

A tabela 1, apresenta informações sobre as comparações eletromiográficas entre os músculos em cada posição dos pés. Ao analisar a interferência da PN na ativação dos músculos RF, VM e VL, verificamos diferença significativa entre o RF em comparação com os demais músculos, VM e VL (RF $x$ VM - $p=0,026$; RF $x$ VL $-p=$ $0,019)$. Entre os músculos VM e VL não houve diferenças significativas $(p=0,831)$. $O$ tamanho do efeito mostrou-se pequeno $(d=0,006)$.

Na posição dos pés em RI, identificamos diferença significativa entre os músculos $R F \times V M(p=0,016)$, e RF $x \operatorname{VL}(p=0,026)$. Entre o VM $x \mathrm{VL}$, não houve diferença significativa $(p=0,534)$. Ao analisar o resultado do tamanho do efeito, o mesmo, apresentou-se pequeno $(d=0,005)$.

Na posição RE não identificamos diferença significativa entre os músculos RF $x$ VM $(p=0,155), \operatorname{RF} \times \operatorname{VL}(p=0,110)$ e VM $x$ VL $(p=0,790)$. O tamanho do efeito nessa análise apresentou a mesma tendência das demais, pequeno $(d=0,003)$. 
Tabela 1.

Comparação da ativação eletromiográfica entre os músculos, nas diferentes posições dos pés.

\begin{tabular}{|c|c|c|c|c|c|c|c|}
\hline & \multicolumn{3}{|c|}{ Músculos } & \multicolumn{3}{c|}{ p } & d \\
\hline Posições & RF $(\mu \mathrm{V})$ & VM $(\mu \mathrm{V})$ & VL $(\mu \mathrm{V})$ & $\begin{array}{c}\text { RF x } \\
\text { VM }\end{array}$ & $\begin{array}{c}\text { RF x } \\
\text { VL }\end{array}$ & $\begin{array}{c}\text { VM x } \\
\text { VL }\end{array}$ & \\
\hline \multirow{2}{*}{ PN $(\mu \mathrm{V})$} & $117,64 \pm$ & $141,55 \pm$ & $148,13 \pm$ & 0,026 & 0,019 & 0,831 & 0,00 \\
& 44,26 & $53,72^{*}$ & $49,02^{* *}$ & & & & 6 \\
\hline \multirow{2}{*}{$\mathrm{RI}(\mu \mathrm{V})$} & $116,56 \pm$ & $142,97 \pm$ & $150,28 \pm$ & 0,016 & 0,026 & 0,534 & 0,00 \\
& 44,81 & $57,73^{* * *}$ & $49,24^{* * * *}$ & & & & 5 \\
\hline \multirow{2}{*}{$\mathrm{RE}(\mu \mathrm{V})$} & $124,83 \pm$ & $143,62 \pm$ & $144,71 \pm$ & 0,155 & 0,110 & 0,790 & 0,00 \\
& 39,75 & 46,87 & 47,43 & & & & 3 \\
\hline
\end{tabular}

Nota: * diferença significativa entre o RF-VM na posição neutra $p<0,026$; ** diferença significativa entre o RF-VL na posição neutra $p<0,019$. ${ }^{* * *}$ diferença significativa entre o RF-VM na rotação interna $p<0,016$. ${ }^{* * * *}$ diferença significativa entre o RF-VL na rotação interna $p<0,026$. RF: reto femoral; VM: vasto medial; VL: vasto lateral; PN: posição neutra; RI: rotação interna, e PE: rotação externa. Fonte: elaboração própria.

Na figura 1, observa-se a análise do músculo RF nas diferentes posições dos pés, onde não foi identificado uma diferença significativa em PN x RI ( $p=0,998), P N \times R E(p$ $=0,926)$ e RI $x \operatorname{RE}(p=0,904)$. O tamanho do efeito foi pequeno $(d=0,008)$. 


\section{Ativação eletromiogáfica do RF nas 3 posições do pé}

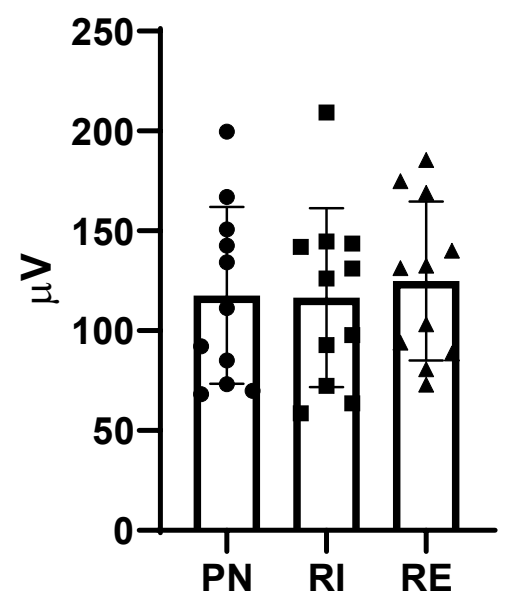

Posições do Pé

Figura 1. Comparação da EMG no músculo RF. Legenda: PN: Posição neutra; RI: Rotação interna, e RE: Rotação externa. Fonte: elaboração própria.

O músculo VM manteve a tendência que o RF, sem diferença significativa entre as posições do pé (PN x RI $p=0,989$; PN x RE $p=0,996$; RI $\times R E p=0,998$ ), com um tamanho do efeito pequeno $(d=0,066)$ (figura 2$)$.

\section{Ativação eletromiogáfica do VM nas 3 posições do pé}

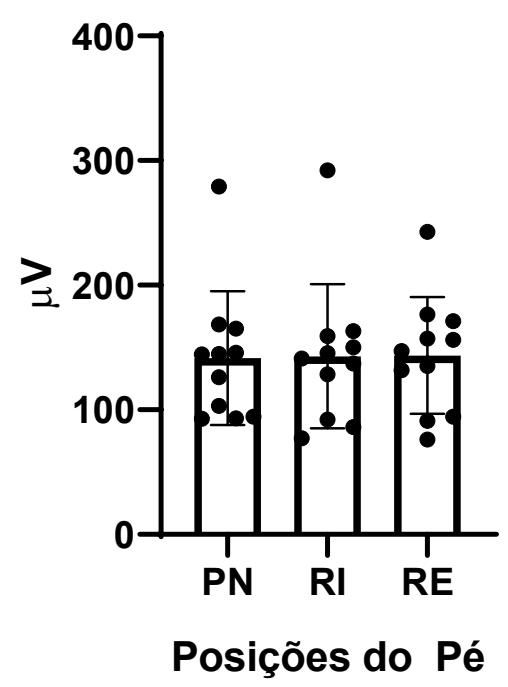

Figura 2. Comparação da EMG no músculo VM. Legenda: PN: Posição neutra; RI: Rotação interna, e RE: Rotação externa. Fonte: elaboração própria.

Na figura 3, refere-se as ativações do músculo $V L$, em que não foi identificado diferença significativa em nenhuma das posições (PN x RI $(p=0,995), P N \times R E(p=$ 
$0,986)$ e RI x RE ( $p=0,965)$ ), o tamanho do efeito se manteve pequeno como nas demais análises $(d=0,002)$.

\section{Ativação eletromiogáfica do VL nas 3 posições do pé}

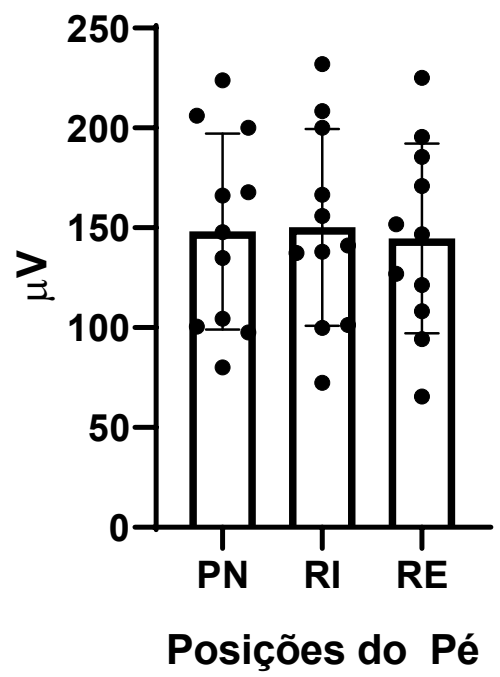

Figura 3. Comparação da EMG no músculo VL. Legenda: PN: Posição neutra; RI: Rotação interna, e RE: Rotação externa. Fonte: elaboração própria.

Ao observar em valores do $\Delta$ de variação da ativação do QF nas diferentes posições dos pés (tabela 3 ), nota-se que a maior variação ocorreu sempre na comparação entre a posição $\mathrm{PN} x \mathrm{RE}$, em todos os músculos analisados.

Tabela 3.

Descrição do $\Delta$ de variação das ativações dos músculos nas diferentes posições dos pés durante a extensão de joelhos.

\begin{tabular}{llll}
\hline Músculos & PN x RI & PN x RE & RI x RE \\
\hline RF & $>0,91 \%$ & $<5,75 \%$ & $<6,62 \%$ \\
VM & $<0,98 \%$ & $<1,44 \%$ & $<0,45 \%$ \\
VL & $<1,42 \%$ & $>2,3 \%$ & $>3,69 \%$
\end{tabular}

Nota: RF: reto femoral; VM: vasto medial; VL: vasto lateral; PN: posição neutra; RI: rotação interna, e RE: rotação externa. Fonte: elaboração própria. 


\section{DISCUSSÃO}

Ao analisar a interferência das diferentes variações na posição dos pés na ativação dos músculos do QF, no movimento de extensão de joelhos, os resultados da presente pesquisa, sugerem que o RF, foi o músculo que demostrou menor ativação em duas, das três posições analisadas.

Verificou-se também, que não houve distinções em relação a uma maior ativação entre os músculos VL e VM. Diante desta análise, entende-se que não seja necessário, alterar a posição dos pés em RI e RE, durante o exercício de cadeira extensora, objetivando maiores ações dos músculos VL e VM.

Estes resultados corroboram parcialmente com os resultados de (Lanza et al., 2017; Saito \& Akima, 2015), que investigaram a relação entre as posições dos pés (PN, RI e RE) e a ativação dos músculos VM e VL, em contrações voluntárias isométricas máximas na extensão dos joelhos. Os autores citados, não observaram diferenças significativas $(p<0,05)$ entre os músculos analisados e sugerem que as diferentes posições dos pés não influenciam na ativação seletiva dos músculos do QF Visscher et al., (2017) e Wu, et al., (2017), também não observaram alterações significativas no nível de ativação eletromiográfica durante as diferentes posições dos pés, em extensão isométrica de joelhos. Em estudos que avaliaram apenas exercícios de cadeia cinética fechada, como o agachamento em diferentes posições dos pés (PN, RI, PE e um ligeiramente à frente do outro), também não encontraram diferenças significativas ( $p<$ 0,05), na atividade elétrica dos músculos analisados (Ando et al., 2018; da Silva et al., 2016).

Em uma pesquisa que objetivou verificar a possível ligação entre as fases do ciclo menstrual e as ativações musculares através da eletromiografia, no agachamento guiado, mantendo a posição neutra dos pés, não se observou diferenças significativas na ativação dos músculos do QF (RF, VM e VL) ( De Lima Costa, Santos, Duarte Rocha \& Da Silva, 2015). Em contrapartida, o presente estudo identificou uma menor ativação do RF na PN dos pés, o que pode ser justificado pela diferença dos exercícios utilizados nas duas pesquisas, sendo um de cadeia cinética aberta e o outro de cadeia cinética fechada. Outro ponto a ser discutido em relação aos achados das diferentes pesquisas, é a arquitetura muscular do QF, interfere de forma distinta na execução dos movimentos realizado na cadeira extensora e agachamento guiado (Ando et al., 2018).

Esperava-se encontrar ativações significativamente maiores para o músculo VL, durante a RI dos pés. Nota-se que, o VM e VL apresentaram valores maiores, durante a PN e a RI, sendo que as maiores ativações foram observadas no VM e no VL. A literatura aponta e justifica que a maior ativação desses músculos durante a RI, devese à maior tensão de estiramento das fibras musculares, que correm obliquamente da 
lateral para a medial do músculo, levando à maior magnitude de ativação (Murray et al., 2013; Visscher et al., 2017). Em uma linha mais antiga e contrária, outros grupos de investigação não reportam diferenças significativas $(p<0,05)$ na ativação elétrica do VL durante as condições de rotações dos pés (Alkner, Tesch \& Berg, 2000; Signorile et al., 1995).

A hipótese de que uma maior magnitude de ativação do RF seria encontrada durante a PN do exercício também não foi sustentada pelo presente estudo, pois o RF apresentou menor ativação nessa posição. Os estudos supracitados observaram que o RF apresentou níveis de ativação muscular maior durante a RE (Alkner et al., 2000; Signorile et al., 1995; Stoutenberg et al., 2005), o que contrapõe com os resultados deste estudo. Essa maior ativação pode ser justificada devido ao seu ponto de origem e estrutura biarticular, diferente dos vastos, enquanto a RE do fêmur pode aumentar a tensão e, por conseguinte, o nível de ativação deste músculo (Signorile et al., 1995). Outros pesquisadores também relataram maior ativação elétrica do RF em condições de RE, durante exercício dinâmico (Alkner et al., 2000; Stoutenberg et al., 2005). Este achado não suporta os resultados do presente estudo, pois não foi encontrada uma ativação diferente na RE. A justificativa que sustenta estes achados está baseada no fato de que o exercício cadeira extensora é monoarticular e isso faz com que os músculos do QF sejam ativados de maneira similar, o que não ocorre quando são usado exercícios multiarticulares, como o agachamento (Akima et al., 2019).

Em adição, o VM também não apresentou diferenças significativas durante as condições neutras e de rotação (de Souza et al., 2018), relatam altos níveis de ativação do VM durante a RE, enquanto uma outra pesquisa relata achados contrários, em que que se observam maiores ativações durante a RI (Stoutenberg et al., 2005).

Com relação às posições dos pés, não foram encontradas diferenças significativas no presente estudo (gráficos 1, 2 e 3). No entanto, destaca-se uma tendência de maior ativação durante a RE em relação à PN e RI. Em contrapartida, são observados maiores níveis de ativação elétrica da PN e RI em relação à RE, durante a contração isométrica, apresentando maior atividade significante $(p=0,013)$ (Akima et al., 2019; Watanabe \& Akima, 2009).

\section{LIMITAÇÕES}

Fatores como as diferenças entre contrações dinâmicas e isométricas e, variações na magnitude de ativação através da mudança de ângulo do joelho, podem justificar a divergência dos resultados (Ando et al., 2018). Condições limitantes do presente estudo podem ser descritas primeiramente, ao fato de ângulo do movimento durante a execução do exercício dinâmico na cadeira extensora não ter sido controlado, o que 
pode permitir a ausência de significância entre as posições dos pés. Em adição, sugerese que a ativação muscular eletromiográfica seja examinada separadamente, durante as fases concêntricas e excêntricas do movimento, para verificar possíveis diferenças de atividade eletromiográfica para estas condições.

\section{CONCLUSÃO}

Em conclusão, os resultados do presente estudo sugerem que as variações nas rotações dos pés - interna ou externamente - durante a extensão de joelhos, na medida em que foram aplicados, não são eficazes para a seleção específica dos músculos que compõem o quadríceps femoral. Estes resultados demonstram que para as aplicações clínicas, as diferentes posições dos pés, na medida em que foram aplicados, não são suficientemente eficazes para aumentar seletivamente o desempenho de músculos do quadríceps, as condições ativam igualmente os músculos do quadríceps, não sendo necessárias variações nas rotações dos pés com o objetivo de manter em equilíbrio a tensão nos músculos e ligamentos responsáveis pelo movimento.

\section{RESPONSABILIDADES ÉTICAS}

Proteção de pessoas e animais. Os autores declaram que os procedimentos seguidos estavam de acordo com os regulamentos estabelecidos pelos responsáveis da Comissão de Investigação Clínica e ética e de acordo com os da Associação Médica Mundial e da Declaração de Helsinki.

Confidencialidade dos dados. Os autores declaram que não aparecem dados de pacientes neste artigo.

Direito à privacidade e consentimento escrito. Os autores declaram que não aparecem dados de pacientes neste artigo.

\section{Conflito de interesses}

Os autores declaram não haver conflito de interesses. 


\section{REFERÊNCIAS}

Akima, H., \& Ando, R. (2017). Oxygenation and neuromuscular activation of the quadriceps femoris including the vastus intermedius during a fatiguing contraction. Clinical Physiology and Functional Imaging, 37(6), 750-758. doi: https://doi.org/10.1111/cpf.12370

Akima, H., Tomita, A., \& Ando, R. (2019). Effect of knee joint angle on the neuromuscular activation of the quadriceps femoris during repetitive fatiguing contractions. Journal of Electromyography and Kinesiology, 49, 102356. doi: https://doi.org/10.1016/j.jelekin.2019.102356

Alkner, B. A., Tesch, P. A., \& Berg, H. E. (2000). Quadriceps EMG/force relationship in knee extension and leg press. Medicine and Science in Sports and Exercise, 32(2), 459-463. doi: https://doi.org/10.1097/00005768-200002000-00030

Ando, R., Nosaka, K., Inami, T., Tomita, A., Watanabe, K., Blazevich, A. J., \& Akima, H. (2016). Difference in fascicle behaviors between superficial and deep quadriceps muscles during isometric contractions. Muscle \& Nerve, 53(5), 797-802. doi: https://doi.org/10.1002/mus.24905

Ando, R., Tomita, A., Watanabe, K., \& Akima, H. (2018). Knee joint angle and vasti muscle electromyograms during fatiguing contractions. Clinical Physiology and Functional Imaging, 38(4), 566-572. doi: https://doi.org/10.1111/cpf.12372

Briani, R. V., De Oliveira Silva, D., Flóride, C. S., Aragão, F. A., De Albuquerque, C. E., Magalhães, F. H., \& De Azevedo, F. M. (2018). Quadriceps neuromuscular function in women with patellofemoral pain: Influences of the type of the task and the level of pain. PLoS ONE, 13(10), e0205553. doi: https://doi.org/10.1371/journal.pone.0205553

Cha, Y.J. (2014). Isokinetic training effect of ankle positions on knee extensor strength. Journal of Physical Therapy Science, 26(9), 1465-1467. doi: https://doi.org/10.1589/jpts.26.1465

Fagan, V., \& Delahunt, E. (2008). Patellofemoral pain syndrome: a review on the associated neuromuscular deficits and current treatment options. British Journal of Sports Medicine, 42(10), 789-795. doi: https://doi.org/10.1136/bjsm.2008.046623

Lanza, M. B., Balshaw, T. G., \& Folland, J. P. (2017). Do changes in neuromuscular activation contribute to the knee extensor angle-torque relationship? Experimental Physiology, 102(8), 962-973. doi: https://doi.org/10.1113/EP086343

De Lima Costa, P., Santos, Pereira F., Duarte Rocha, C.C., Da Silva, F. S. (2015). Study of the electromyographic activation of lower limbs during the menstrual cycle phases. Journal of Exercise Physiology Online, 18(2), 52-57. Recuperado de https://bit.ly/3m4vy6Y 
Hermens, H.J., Merletti, R., Freiks, B. (1996). European Activities on Surface Electromyography. In Proceedings of the first general SENIAN workshop. Enschede, Netherlands: Publishers Roessing Research and Development. Recuperado de http://www.seniam.org/pdf/contents1.PDF

Murray, N., Cipriani, D., O'Rand, D., \& Reed-Jones, R. (2013). Effects of Foot Position during Squatting on the Quadriceps Femoris: An Electromyographic Study. International Journal of Exercise Science, 6(2), 114-125. Recuperado de http://www.ncbi.nIm.nih.gov/pubmed/27293497

Noorkõiv, M., Nosaka, K., \& Blazevich, A. J. (2014). Neuromuscular adaptations associated with knee joint angle-specific force change. Medicine and Science in Sports and Exercise, 46(8), 1525-1537. doi: https://doi.org/10.1249/MSS.0000000000000269

Saito, A., \& Akima, H. (2015). Neuromuscular Activation of the Vastus Intermedius Muscle during Isometric Hip Flexion. PLOS ONE, 10(10), e0141146. doi: https://doi.org/10.1371/journal.pone.0141146

Signorile, J. F., Kwiatkowski, K., Caruso, J. F., \& Robertson, B. (1995). Effect of foot position on the electromyographical activity of the superficial quadriceps muscles during the parallel squat and knee extension. Journal of Strength and Conditioning Research, 9(3), 182-187. doi: https://doi.org/10.1519/00124278-199508000$\underline{00011}$

da Silva, C. R., de Oliveira Silva, D., Briani, R. V., Pazzinatto, M. F., Ferrari, D., \& de Azevedo, F. M. (2016). Test-retest reliability of electromyographic signal parameters used to evaluate neuromuscular fatigue in quadriceps femoris muscle. Kinesiology, 48(2), 174-181. doi: https://doi.org/10.26582/k.48.2.14

de Souza, L. M. L., Cabral, H. V., de Oliveira, L. F., \& Vieira, T. M. (2018). Motor units in vastus lateralis and in different vastus medialis regions show different firing properties during low-level, isometric knee extension contraction. Human Movement Science, 58, 307-314. doi: https://doi.org/10.1016/j.humov.2017.12.012

Spairani, L., Barbero, M., Cescon, C., Combi, F., Gemelli, T., Giovanetti, G., Magnani, B., \& D'Antona, G. (2012). An electromyographic study of the vastii muscles during open and closed kinetic chain submaximal isometric exercises. International Journal of Sports Physical Therapy, 7(6), 617-626. Recuperado de https://www.ncbi.nlm.nih.gov/pmc/articles/PMC3537462/

Stoutenberg, M., Pluchino, A. P., Ma, F., Hoctor, J. E., \& Signorile, J. F. (2005). The impact of foot position on electromyographical activity of the superficial quadriceps 
muscles during leg extension. Journal of Strength and Conditioning Research, 19(4), 931-938. Recuperado de http://www.ncbi.nlm.nih.gov/pubmed/16437818

Visscher, R. M. S., Rossi, D., Friesenbichler, B., Dohm-Acker, M., Rosenheck, T., \& Maffiuletti, N. A. (2017). Vastus medialis and lateralis activity during voluntary and stimulated contractions. Muscle \& Nerve, 56(5), 968-974. doi: https://doi.org/10.1002/mus.25542

Watanabe, K., \& Akima, H. (2009). Normalized EMG to normalized torque relationship of vastus intermedius muscle during isometric knee extension. European Journal of Applied Physiology, 106(5), 665-673. doi: https://doi.org/10.1007/s00421-0091064-z

Wu, R., Delahunt, E., Ditroilo, M., Lowery, M. M., \& De Vito, G. (2017). Effect of Knee Joint Angle and Contraction Intensity on Hamstrings Coactivation. Medicine \& Science in Sports \& Exercise, 49(8), 1668-1676. doi: https://doi.org/10.1249/MSS.0000000000001273 